\title{
Firing up ambition
}

\section{Commitment to climate action is needed in the coming year to avoid further impacts such as the wildfires that have grabbed headlines in 2019.}

10 ope, ambition, youth and despair are all topics that have been linked to climate change in the popular media in recent months. Reflecting on climate action in 2019, there was a surge in youth engagement, and public engagement more generally, with the Fridays for Future movement, and climate strikes expanding around the globe. September saw New York host the UN Climate Summit, an event that set the scene for engagement and action in advance of the 25th Conference of the Parties (COP25) to the UN Framework Convention on Climate Change, which at the time of writing had overrun the expected timeline and just concluded in Madrid, with host Chile promoting the increase of overall ambition on climate action from governments, alongside greater inclusion of indigenous people, gender aspects, and loss and damage, among others. Discussions were focused on completing some of the working aspects of the Paris Agreement to allow it to be fully operational, with Article 6 — international climate markets - needing to be finalized and agreed.

Alongside the climate negotiations and actions are the impacts of climate change that are already being felt. Again, the devastation of wildfires is affecting many around the world. Recent images from the east coast of Australia, including the satellite images showing the expansive stretch of land burning, and the smoke haze covering Sydney, are a stark reminder of the dangers that the fires pose to property and life. Wildfires are now a perennial issue, and one to which this journal keeps returning, given the immediate and largescale impact which can trigger strong emotional response. Concerns were raised in our November 2017 editorial (https://doi. org/10.1038/nclimate3432) that the wildfires of that year were a sign of things to come; and our December 2018 editorial (https://doi.org/10.1038/s41558-018-0369-5) emphasized that the year's wildfires had reinforced the concerns.

2019 did nothing to assuage those concerns, with the current Australian fires, October's fires in California that necessitated power cuts to avoid sparks igniting further blazes (PGE, https://go.nature. com/2LLQcbO; 2019), and the widespread blazes across the Amazon rainforest during the dry season being some of the larger

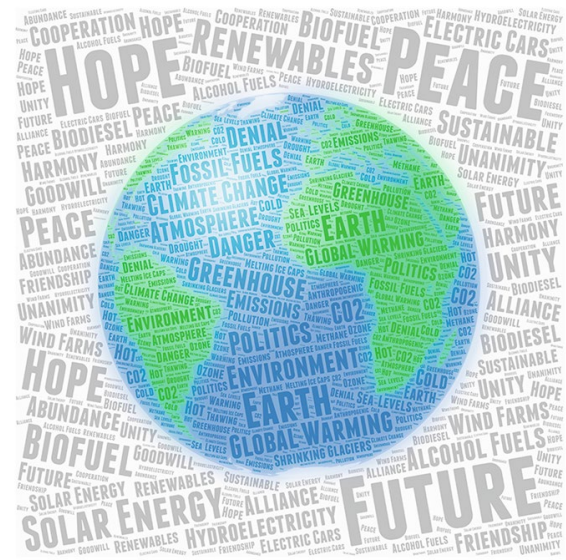

Credit: Mark Garlick/Science Photo Library / Science Photo Library / Getty

events. In the Amazon, August 2019 had nearly three times the number of fires seen in August 2018 (and the highest since 2010), apparently linked to higher deforestation (Glob. Change Biol. https://doi.org/10.1111/ gcb.14872; 2019). These events have stood out and attracted widespread media coverage, but there were events spread around the globe, with some areas, such as these mentioned, seeing higher fire incidents and intensity, while other areas had lower fire activity than previous years (see https://atmosphere. copernicus.eu/did-2019-really-bring-usunusual-number-wildfires for summary of the full year, including visualization of wildfires around the world in 2019).

The increase in the size and scale of events, combined with longer fire seasons, means that the international community of firefighters is stretched to the limit with finite resources and budgets, and the possibility of exchange of manpower between Northern and Southern Hemisphere is reduced. Yet despite these devastating events, there is a lack of political response from some governments and leaders, including those in affected areas. In Brazil, there has been denial that the fires were outside the norm for the dry season (the reported motivator behind the Global Change Biology publication cited above, as the authors wanted to refute those statements). Australia is claiming to be on target for emission reductions, although use of carryover credits - from overachieving on the promised Kyoto Protocol reductions is an accounting loophole not really in the spirit of increased ambition (M. Foley, Sydney Morning Herald, https://go.nature. com/38quXps; 2019). Whether the outcomes of the COP25 negotiations will influence these, and other, governments towards greater ambition is unclear, but optimism in the immediate wake of the event is low.

But what will 2020 bring? In the political and climate action space, many countries are already conscientiously working towards their new national climate action plans, known as nationally determined contributions (NDCs), to be submitted in advance of COP26 in November 2020. Globally, ambition needs to increase, and whereas the first intended NDCs (which became many countries' committed NDCs) were produced on a tight timeframe, the discussion around revised NDCs shows greater inclusion of sectors to provide a more holistic approach rather than being mitigation-centric, and further integration into domestic policy. The disappointment on the lack of agreement around Article 6, among other areas of limited progress, puts greater pressure on COP26 to reignite the ambitious spirit that came out of Paris four years ago.

Additionally, in November 2019, the United States of America announced their intention to withdraw from the Paris Agreement, with this coming into effect in November 2020 if it proceeds. What this means is uncertain, although an opinion piece we published in May 2017 (L. Kemp, Nat. Clim. Change 7, 458-460) suggests that the agreement might be stronger once they leave.

Although COP26, to be held in Glasgow, UK, will be the pinnacle event for climate negotiations, there are also international negotiations under way for a global biodiversity framework (https://www.cbd. int/conferences/post2020). The parties to the Convention on Biological Diversity will meet in October to agree the post-2020 framework, which will look ahead to 2050 in order to meet the vision of "Living in harmony with nature". Success in these international agreements requires global engagement and commitment to ensure a planet for all.

Published online: 2 January 2020 https://doi.org/10.1038/s41558-019-0680-9 